\title{
Preliminary Take-Off Analysis and Simulation of PrandtIPlane Commercial Aircraft
}

\author{
K. Abu Salem ${ }^{1} \cdot$ G. Palaia ${ }^{1} \cdot$ M. Bianchi ${ }^{1} \cdot$ D. Zanetti ${ }^{2} \cdot$ V. Cipolla ${ }^{1} \cdot$ V. Binante ${ }^{2}$ \\ Received: 17 February 2020 / Revised: 9 July 2020 / Accepted: 13 July 2020 / Published online: 21 July 2020 \\ (c) The Author(s) 2020
}

\begin{abstract}
The present paper deals with the take-off performance analysis of PrandtlPlane aircraft. The PrandtlPlane is a Box-Wing configuration based on Prandtl's "Best Wing System" concept, which minimizes the induced drag once wingspan and lift are given. The take-off dynamics is simulated implementing the non-linear equations of motion in a numerical tool, which adopts a Vortex Lattice Method solver to evaluate the aerodynamics characteristics taking also ground effects into account. The take-off analysis is performed for both a PrandtlPlane and a reference monoplane, with the aim of comparing the performance of the two different architectures. The preliminary results show the potential advantages of the PrandtlPlane, such as runway length reduction and improved passenger comfort.
\end{abstract}

Keywords PrandtlPlane $\cdot$ Box-Wing $\cdot$ Take-off $\cdot$ PARSIFAL

\section{List of Symbols}

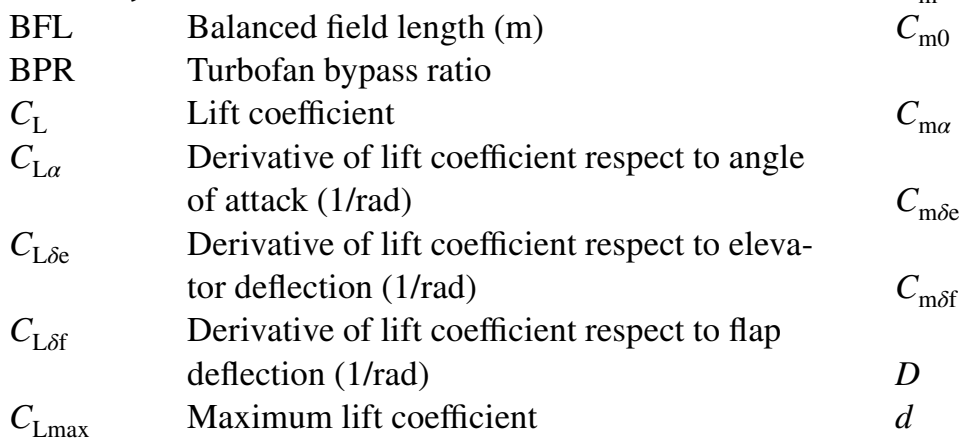

$\triangle$ K. Abu Salem

karim.abusalem@gmail.com

G. Palaia

giuseppe.palaia@phd.unipi.it

M. Bianchi

bianchigino@inwind.it

D. Zanetti

d.zanetti@skyboxeng.com

V. Cipolla

vittorio.cipolla@unipi.it

V. Binante

v.binante@skyboxeng.com

1 Department of Civil and Industrial Engineering, University of Pisa, Pisa, Italy

2 SkyBox Engineering S.r.l., Pisa, Italy

\begin{tabular}{|c|c|}
\hline$C_{\mathrm{m}}$ & Pitching-moment coefficient \\
\hline$C_{\mathrm{m} 0}$ & $\begin{array}{l}\text { Pitching-moment coefficient at zero angle of } \\
\text { attack }\end{array}$ \\
\hline$C_{\mathrm{m} \alpha}$ & $\begin{array}{l}\text { Derivative of pitching moment coefficient } \\
\text { respect to angle of attack }(1 / \mathrm{rad})\end{array}$ \\
\hline$C_{\mathrm{m} \delta \mathrm{e}}$ & $\begin{array}{l}\text { Derivative of pitching moment coefficient } \\
\text { respect to elevator deflection }(1 / \mathrm{rad})\end{array}$ \\
\hline$C_{\mathrm{m} \delta \mathrm{f}}$ & $\begin{array}{l}\text { Derivative of pitching moment coefficient } \\
\text { respect to flap deflection }(1 / \mathrm{rad})\end{array}$ \\
\hline$D$ & $\operatorname{Drag}(\mathrm{N})$ \\
\hline$d$ & $\begin{array}{l}\text { Centre of gravity-wheels horizontal distance } \\
\text { (m) }\end{array}$ \\
\hline$g$ & Standard gravity $\left(\mathrm{m} / \mathrm{s}^{2}\right)$ \\
\hline$h$ & Centre of gravity-wheels vertical distance (m) \\
\hline$I_{\mathrm{Y}}$ & Pitching moment of inertia $\left(\mathrm{kgm}^{2}\right)$ \\
\hline$L$ & $\operatorname{Lift}(\mathrm{N})$ \\
\hline$M_{\mathrm{A}}$ & Aerodynamic pitching moment $(\mathrm{N} \mathrm{m})$ \\
\hline MTOW & Maximum take-off weight $(\mathrm{N})$ \\
\hline$n_{\mathrm{Z}}$ & Vertical load factor \\
\hline$R_{\mathrm{N}}$ & Vertical reaction of the ground $(\mathrm{N})$ \\
\hline$R_{\mathrm{T}}$ & Horizontal reaction of the ground $(\mathrm{N})$ \\
\hline$T$ & Thrust $(\mathrm{N})$ \\
\hline TOD & Take-off distance (m) \\
\hline$V$ & Speed $(\mathrm{m} / \mathrm{s})$ \\
\hline$V_{1}$ & Decision speed $(\mathrm{m} / \mathrm{s})$ \\
\hline$V_{2}$ & Take-off speed (m/s) \\
\hline$V_{\mathrm{R}}$ & Rotation speed (m/s) \\
\hline$V_{\mathrm{Z}}$ & Vertical speed (m/s) \\
\hline
\end{tabular}




$\begin{array}{ll}W & \text { Aircraft weight }(\mathrm{N}) \\ \left(X_{\mathrm{F}} Y_{\mathrm{F}} Z_{\mathrm{F}}\right) & \text { Final coordinates of main surfaces }(\mathrm{m}) \\ \left(X_{\mathrm{I}} Y_{\mathrm{I}} Z_{\mathrm{I}}\right) & \text { Initial coordinates of main surfaces }(\mathrm{m}) \\ \alpha & \text { Angle of attack }(\mathrm{rad}) \\ \gamma & \text { Trajectory angle }(\mathrm{rad}) \\ \Delta_{\mathrm{Z}} & \text { Height }(\mathrm{m}) \\ \delta_{\mathrm{e}} & \text { Deflection angle of elevators }(\mathrm{rad}) \\ \delta_{\mathrm{f}} & \text { Deflection angle of flap (rad) } \\ \theta & \text { Attitude angle (rad) } \\ \mu & \text { Rolling friction coefficient } \\ \Psi_{\mathrm{f}} & \text { Flap gain }\end{array}$

\section{Introduction}

The aviation demand growth is around $4.5 \%$ per year $[1,2]$, and it is foreseen to double in a couple of decades [3]. If on one hand these trends indicated a big market opportunity for aviation business, on the other hand they raise a warning in term of negative impact on the environment and service congestion $[4,5]$. The requirements given by ACARE [6] of cutting the $\mathrm{CO}_{2}$ and $\mathrm{NO}_{X}$ pollutions and reducing significantly the external noise around the airport areas will be hardly satisfied without applying deep changes in state-ofthe-art aircraft technology. Among the envisaged innovation strategies, the adoption of non-conventional aircraft configurations provided with higher aerodynamic efficiency is considered as a possible solution to seize the market opportunity while meeting the environmental requirements.

Different innovative aerodynamic configurations [7] have been proposed for the civil aviation of the future, such as the Blended Wing Body [8], the Strut Braced Wings [9] and the Box-Wing [10]. According to 1920s studies due to Ludwig Prandtl's [11], the Box-Wing configuration minimizes the induced drag among all the possible lifting systems with the same total lift and the same span. Prandtl's studies have inspired several research, focused on both the theoretical aspects [12-16] and the application of such concept to different aircraft categories, ranging from ultralight aircraft to transport aircraft with payload capabilities beyond present wide-bodies [17-19].

Among these, studies started in the 1990s at the University of Pisa on the so-called "PrandtlPlane" configuration (PrP hereafter), have led to the research project PARSIFAL (Prandtlplane ARchitecture for the Sustainable Improvement of Future AirpLanes), funded under the Horizon 2020 Program of European Union in 2017. An artistic representation of the PrP object of study in the project is given in Fig. 1.

The PARSIFAL project aims to assess the impact of adopting the Box-Wing configuration from the environmental, economic and logistic standpoints. In particular, the project's goal is to demonstrate that the application of

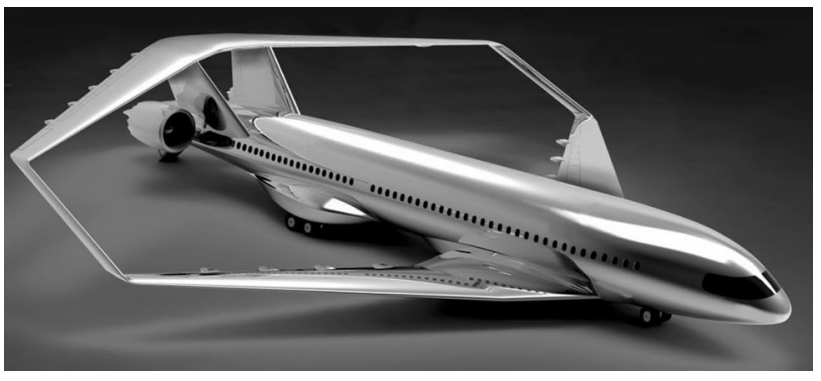

Fig. 1 Artistic representation of the PrandtlPlane object of study in the project PARSIFAL

the PrandtlPlane configuration to aircraft with the same overall dimensions of conventional short-to-medium range aircraft, in particular those with the wingspan between 24 and 36 metres (hence compliant to ICAO Aerodrome Reference Code "C"), can increase the passenger number from about 200 of today conventional aircraft (e.g.: Airbus A320 or Boeing 737) up to more than 300 passengers [20], hence with a significant reduction of environmental impact and direct costs per passenger.

In this context, the study and the analysis of take-off performance of a new aircraft architecture are of primary relevance. In addition, it is well known from the literature $[21,22]$ that during the take-off phase the aircraft is subject to the ground effect, which improves the aerodynamic performance. For the PrP, the ground effect can influence the aerodynamic characteristics in take-off more than a conventional aircraft, since the front wing is very close to the runway. Therefore, the goals of the work are:

- to investigate the Box-Wing in ground effect behaviour during take-off manoeuvre;

- to study the influence of design parameters on take-off performance, such as balanced field length and decision speed;

- to perform a comparison between the PrP and conventional reference aircraft.

The paper is organized as follows: in the first part the two aircraft object of study, the PrP and the conventional reference aircraft, are introduced, and a common procedure to define the control surfaces is presented; the second part describes the implementation of the non-linear equations of motion in a numerical tool, which adopts a Vortex Lattice Method solver to evaluate the aerodynamics in ground effect. In the third part, the results of the simulations are presented, focusing on the comparison between the take-off performance of the two aircraft. 


\subsection{Description of Aircraft Configurations}

To compare the effects of adopting the PrP as an alternative solution to present conventional aircraft, the definition of a common reference model representing state-of-the-art aircraft technology is required. To be compliant with the ICAO Aerodrome Reference Code "C" standard, the reference aircraft chosen in the PARSIFAL project is the CeRAS CSR-01 [23-25], a public reference model for short-medium range aircraft (Fig. 2).

The PrP configuration here investigated is the result of the first design loop performed in PARSIFAL project, described in details in [26-29] (Fig. 3).

To evaluate and to compare the take-off performance of these two configurations, it has been necessary to define a common sizing procedure for high-lift devices and control surfaces. Indeed, the low-speed performance is strictly related to the design of these components. A preliminary sizing procedure for both elevators and flaps has been defined; the procedure is based on the trim fulfilment in the approach condition. The trim problem is thus defined as follows:

$\left\{\begin{array}{l}C_{\mathrm{L}}=C_{\mathrm{L} \alpha} \alpha+C_{\mathrm{L} \delta \mathrm{e}} \delta_{\mathrm{e}}+C_{\mathrm{L} \delta \mathrm{f}} \delta_{\mathrm{f}} \\ C_{\mathrm{m} 0}+C_{\mathrm{m} \alpha} \alpha+C_{\mathrm{m} \delta \mathrm{e}} \delta_{\mathrm{e}}+C_{\mathrm{m} \delta \mathrm{f}} \delta_{\mathrm{f}}=0 .\end{array}\right.$

For the PrP, there are many possible layouts for positioning the movables; the one selected for the current analyses is represented in Fig. 4 (right): the elevators are placed in the root regions of both wings; the ailerons are installed in the tip regions, and the flaps are placed between the elevators
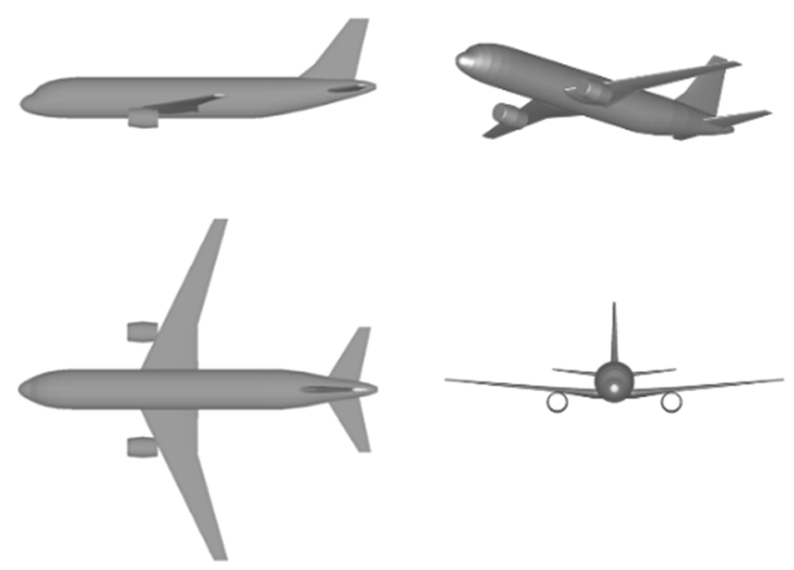

\begin{tabular}{|c|c|c|c|}
\hline Parameter & Symbol & Value & Unit \\
\hline Maximum take-off weight & $W$ & $7,7 \cdot 10^{4}$ & $\mathrm{~kg}$ \\
Moment of inertia & $I_{y}$ & $8,21 \cdot 10^{6}$ & $\mathrm{~kg} \mathrm{~m}^{2}$ \\
Wing surface & $S_{\text {ref }}$ & 122,4 & $\mathrm{~m}^{2}$ \\
Span & $b$ & 34.07 & $\mathrm{~m}$ \\
Mean aerodynamic chord & $\bar{c}$ & 4,18 & $\mathrm{~m}$ \\
Maximum thrust-to-weight ratio & $T_{\max } / W$ & 0,3 & - \\
\hline
\end{tabular}

Fig. 2 Three views and main characteristics of the CeRAS CSR-01 configuration

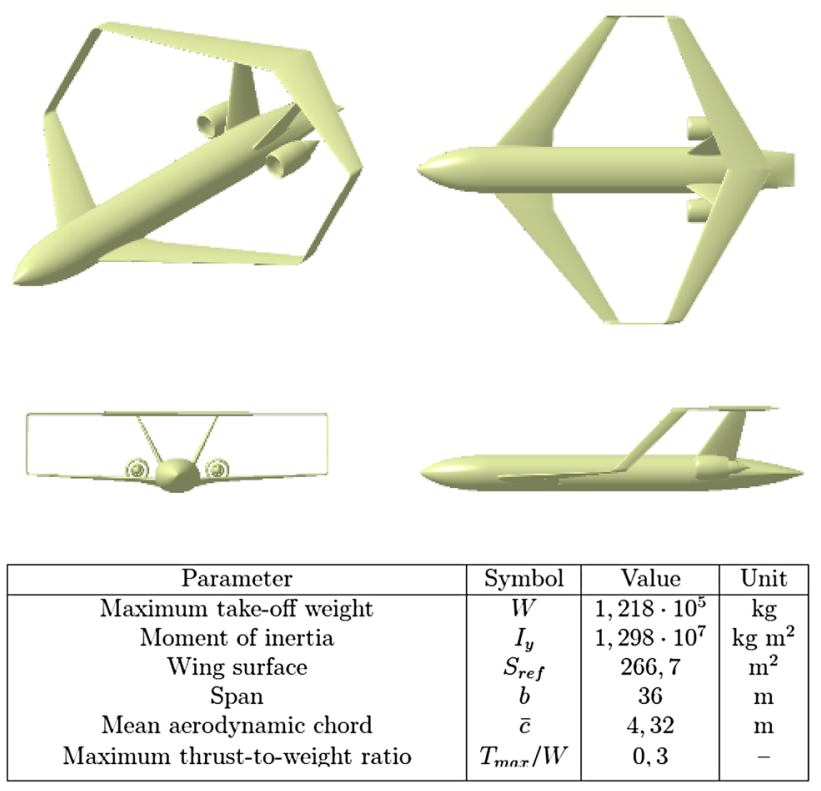

Fig. 3 Three views and main characteristics of the PrP configuration

and the ailerons. Front and rear elevators are actuated with opposite deflections to introduce a pitching moment with minimum variations of total lift. For the reference aircraft, the layout is the conventional one, with the elevators placed on the horizontal tailplane and the flap on the main wing, as sketched in Fig. 4 (left).

The trim problem is solved using the AVL code [30]; the flap deflection is set as an input, and the aerodynamic solver finds $\alpha$ and $\delta_{\mathrm{e}}$ to fulfil vertical and pitch equilibrium in approach condition, at Maximum Landing Weight. All the movables are modelled in AVL as plain flaps, including the high-lift devices. The low-speed performance of the configurations designed with these procedures are estimated by means of consolidated literature methods, as [31]. The whole procedure is schematized in Fig. 5 and described in [26, 32].

\section{Take-Off Simulation Model}

It is possible to divide the take-off manoeuvre into three different segments: ground-roll, rotation, and lift-off, as sketched in Fig. 6. In the ground roll segment, the aircraft starts to accelerate and reaches the rotation speed $V_{\mathrm{R}}$; the only degree of freedom is the longitudinal motion of the aircraft on the runway. The rotation segment starts when the $V_{\mathrm{R}}$ speed is reached and consists in the rotation of the aircraft around the main landing gear; this stage ends when the aircraft pulls the wheels off the ground. Then, in the lift-off phase, the aircraft follows a near-circular path followed by the subsequent climb segment. According to Federal Aviation Regulations (FAR), at the end of the runway the aircraft must reach a minimum height of 
Fig. 4 Movables layout for the two configurations
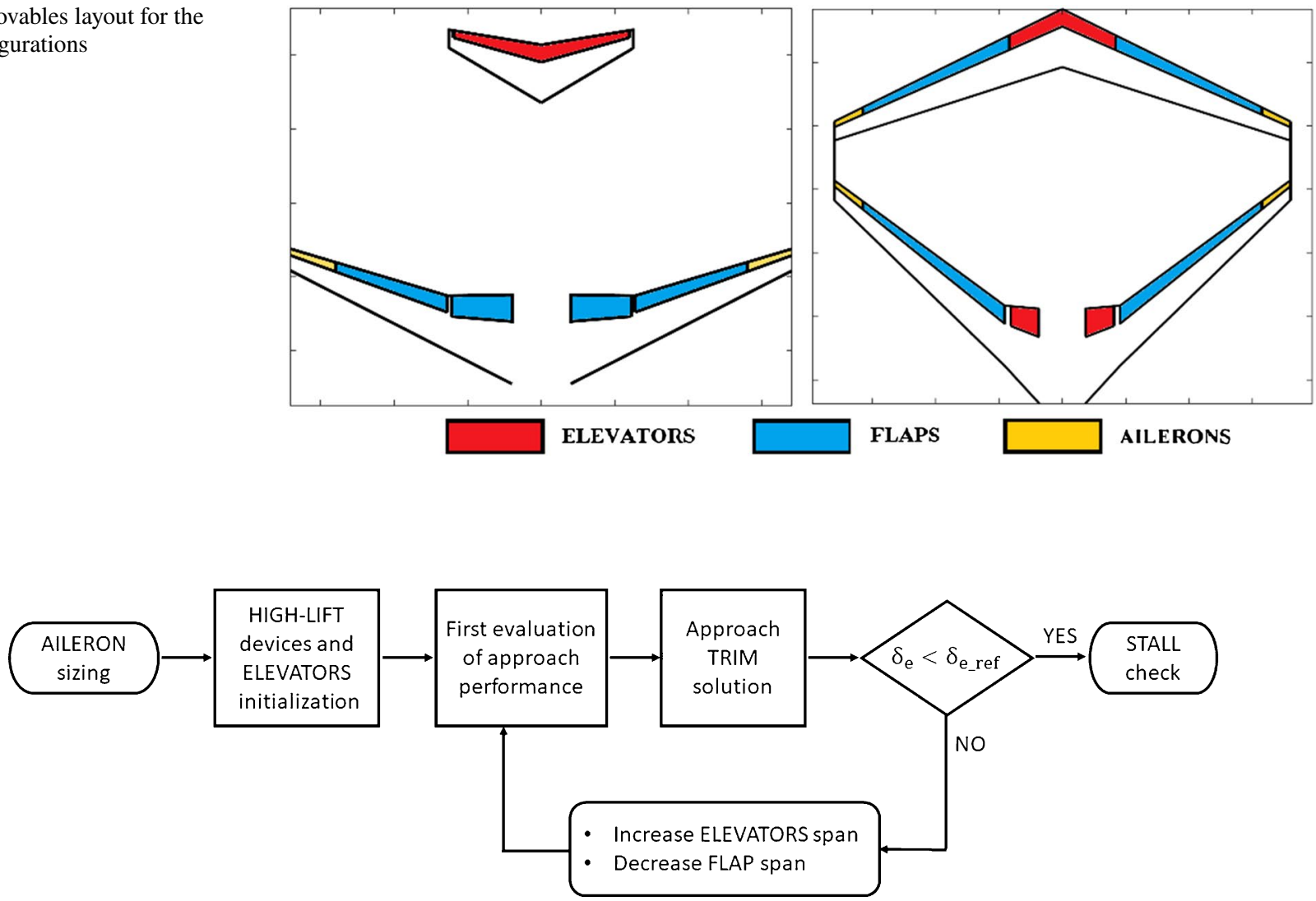

Fig. 5 Preliminary sizing procedure for control surfaces and high-lift devices

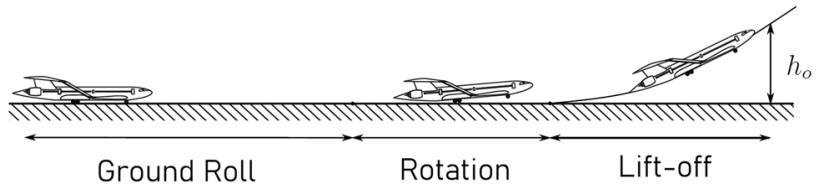

Fig. 6 Take-off manoeuvre

$35 \mathrm{ft}$ and, at that point, its speed $\left(V_{2}\right)$ cannot be lower than 1.2 times the stall speed with flaps extracted.

\subsection{Equations of Motion}

Following the forces schemes for the three take-off phases reported in Fig. 7, the equations of motion can be written as follows:

Ground Roll: $\left\{\begin{array}{l}\frac{W}{g} \frac{\mathrm{d} V}{\mathrm{~d} t}=T-D-R_{\mathrm{T}} \\ R_{\mathrm{N}}+L=W \\ R_{\mathrm{T}}=\mu R_{\mathrm{N}}\end{array}\right.$
Rotation: $\left\{\begin{array}{l}\frac{W}{g} \frac{\mathrm{d} V}{\mathrm{~d} t}=T \cos \theta-D-R_{\mathrm{T}} \frac{W}{g} \frac{\mathrm{d} V}{\mathrm{~d} t}=T \cos \theta-D-R_{\mathrm{T}} \\ I_{\mathrm{y}} \frac{\mathrm{d}^{2} \theta}{\mathrm{d} t^{2}}=M_{\mathrm{A}}-R_{\mathrm{N}} d-R_{\mathrm{T}} h \\ R_{\mathrm{N}}+L+T \sin \theta=W \\ R_{\mathrm{T}}=\mu R_{\mathrm{N}}\end{array}\right.$

Lift off: $\left\{\begin{array}{l}\frac{W}{g} \frac{\mathrm{d} V_{x}}{\mathrm{~d} t}=T \cos \theta-D \cos \gamma-L \sin \gamma \\ \frac{W}{g} \frac{\mathrm{d} V_{z}}{\mathrm{~d} t}=T \sin \theta-D \sin \gamma+L \cos \gamma-W \\ I_{\mathrm{y}} \frac{\mathrm{d}^{2} \theta}{\mathrm{d} t^{2}}=M_{A} .\end{array}\right.$

The differential equations of motion are non-linear, and the degrees of freedom are coupled so that a closed-form solution is not possible; thus, it has been necessary to provide numerical solutions. The integration is conducted with the Euler Method with a time step of $10^{-2} \mathrm{~s}$; due to the implementations of the aerodynamic evaluations in each step of the integration, the method has a high computational cost. 

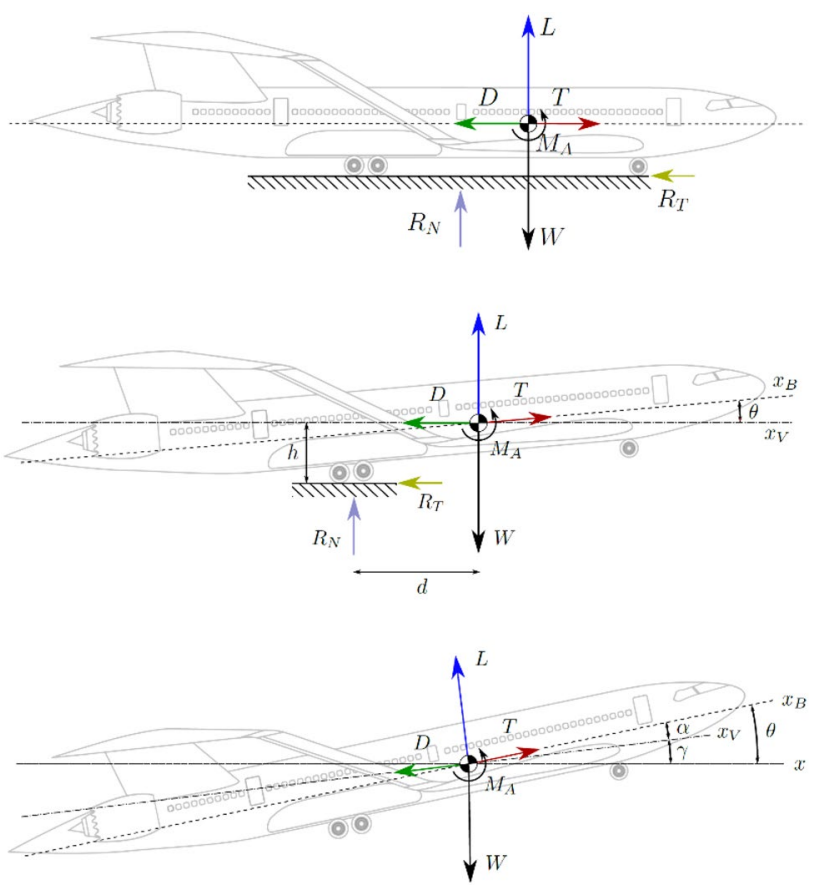

Fig. 7 Forces schemes for the three take-off segments: ground roll (top), rotation (centre), lift off (bottom)

\subsection{Mathematical Model for the Aircraft Geometry Evaluation with Respect to Attitude Variation}

During the ground roll phase, the aircraft attitude does not change, whereas in the rotation and lift-off phases attitude and height change. The main difference between the two last phases is the position of the instant centre of rotation: during the rotation phase, it is the contact point between the tires of the main landing gear and the ground; in the lift-off phase, it is the centre of gravity. The aircraft-ground relative position has to be evaluated accurately to estimate the aerodynamic coefficients in ground effect, so a proper mathematical model has been developed.

\subsubsection{Rotation}

Exceeding the $V_{\mathrm{R}}$ speed, the aircraft deflects the elevator and pitches-up around the ground-tire contact point. The rotation is around an axis parallel to the pitch axis, so there is a symmetry respect to its longitudinal plane, and an easy formulation can be obtained without losing generality. The reference system $(\tau)$ has the origin in the nose of the aircraft and, after the rotation, all coordinates of the main surfaces change. Indicating with $\left[X_{\mathrm{F}}, Y_{\mathrm{F}}, Z_{\mathrm{F}}\right]$ the final coordinates in the reference system and with $\left[X_{\mathrm{I}}, Y_{\mathrm{I}}, Z_{\mathrm{I}}\right]$ the initial coordinates in $\tau$, we obtain:
$\left[\begin{array}{c}X_{\mathrm{F}} \\ Y_{\mathrm{F}} \\ Z_{\mathrm{F}}\end{array}\right]=\left[\begin{array}{ccc}\cos \theta & 0 & \sin \theta \\ 0 & 1 & 0 \\ -\sin \theta & 0 & \cos \theta\end{array}\right]\left[\begin{array}{c}X_{\mathrm{I}}-X_{\mathrm{R}} \\ Y_{\mathrm{I}} \\ Z_{\mathrm{I}}-Z_{\mathrm{R}}\end{array}\right]+\left[\begin{array}{c}X_{\mathrm{R}} \\ 0 \\ Z_{\mathrm{R}}\end{array}\right]$.

The coordinates $X_{\mathrm{R}}$ and $Y_{\mathrm{R}}$ indicate the position of the instant centre of rotation; $\theta$ indicates elevation angle of the aircraft.

\subsubsection{Lift Off}

When the aircraft lifts off from the ground, the centre of gravity becomes the new instant centre of rotation. The equations are similar to the previous ones, but we have to take the relative distance ground-centre of gravity $\left(\Delta_{Z}\right)$ into account:

$\left[\begin{array}{c}X_{\mathrm{F}} \\ Y_{\mathrm{F}} \\ Z_{\mathrm{F}}\end{array}\right]=\left[\begin{array}{ccc}\cos \theta & 0 & \sin \theta \\ 0 & 1 & 0 \\ -\sin \theta & 0 & \cos \theta\end{array}\right]\left[\begin{array}{c}X_{\mathrm{I}}-X_{\mathrm{R}} \\ Y_{\mathrm{I}} \\ Z_{\mathrm{I}}-Z_{\mathrm{R}}\end{array}\right]+\left[\begin{array}{c}X_{\mathrm{R}} \\ 0 \\ Z_{\mathrm{R}}+\Delta_{\mathrm{Z}}\end{array}\right]$.

All these coordinates allow to define the new geometry to evaluate the aerodynamic coefficient in ground effect with AVL solver.

\subsubsection{Evaluation of Aerodynamic Coefficients}

The aerodynamic characteristics of an aircraft during takeoff are strongly influenced by the ground effect, whose main consequences are a significant reduction of the induced drag, due to the modifications of tip vortices and downwash, and an increase of the lift-generating capabilities [21, 22]. The ground effect depends on the aerodynamic characteristics of the aircraft and, in particular, on the clearance of the wings from the ground. For this reason, to realistically simulate the aerodynamics of the aircraft during the take-off phase, it has been decided to evaluate the aerodynamic characteristics in each time step considered; in fact, the position of the aircraft with respect to the ground varies during the evolution of the manoeuvre, depending on the variables $z(t)$ and $\theta(t)$, as described in Sect. 2.2.

Given a large number of aerodynamic evaluations to be done for each simulation, the computation of the aerodynamic characteristics has been carried out using low-fidelity codes, with the aim of limiting the computational time. In particular, the AVL code, based on the Vortex Lattice Method, has been used; with the AVL code, the evaluation of ground effect has been studied by imposing the symmetry boundary condition on the runway plane. A validation procedure to assess the accuracy of AVL when simulating ground effect has been presented in [33], considering as benchmark the results presented in $[34,35]$. The AVL models for the PrP and CeRAS CSR-01 configuration are shown in Fig. 8. 
The main differences between the two configurations that affect the aerodynamics in ground effect consist in the shape of the lifting systems, and the engine position, that influences the ground clearance of the aircraft. In the case of the PrP configuration, the front wing is very close to the runway;

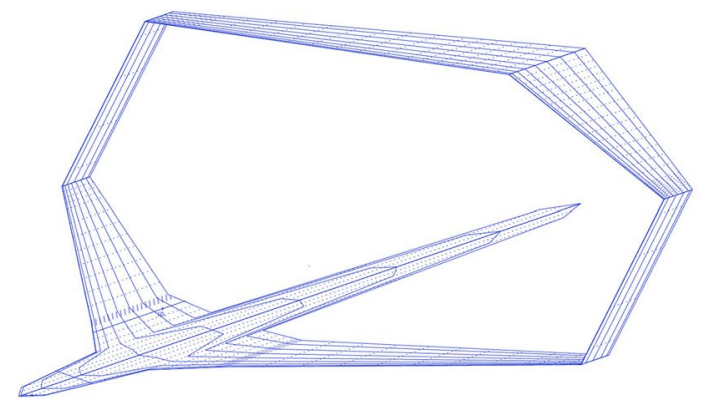

Fig. 8 PrP (left) and CeRAS CSR-01 (right) AVL models

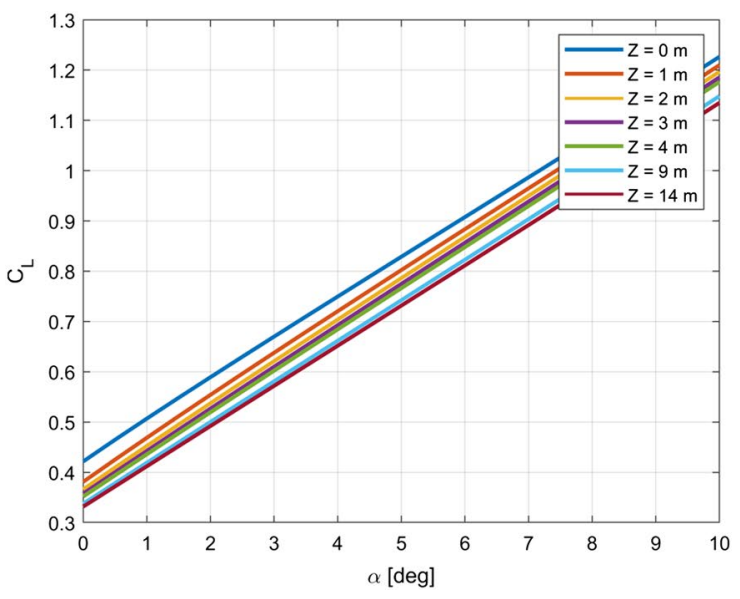

this, because the engines are not placed under the wing, but are positioned in the rear part of the fuselage (see Fig. 1); in the case of a conventional monoplane aircraft, the main wing has a larger distance from the runway, due to the wingmounted engine (see Fig. 2). In the following Figs. 9, 10,

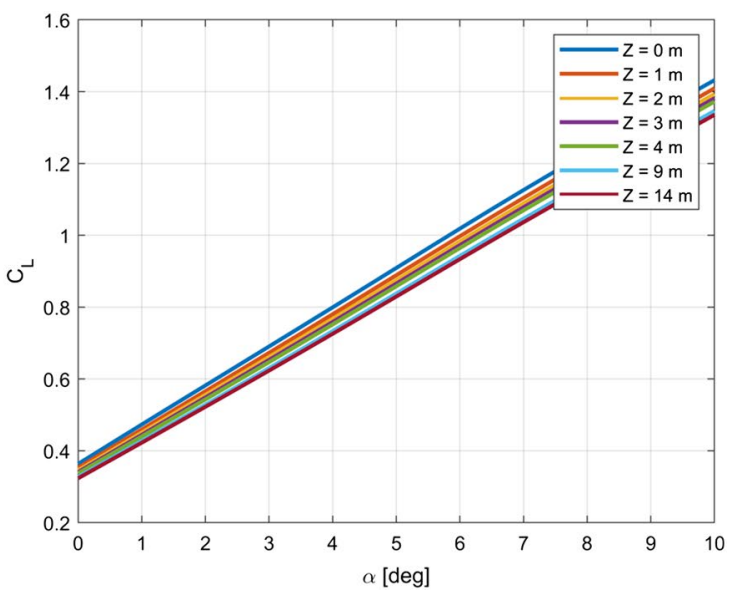

Fig. $9 C_{\mathrm{L}}$ curves for PrP (left) and CeRAS CSR-01 (right) at clean configuration $\left(\delta_{\mathrm{e}}=\delta_{\mathrm{f}}=0\right)$
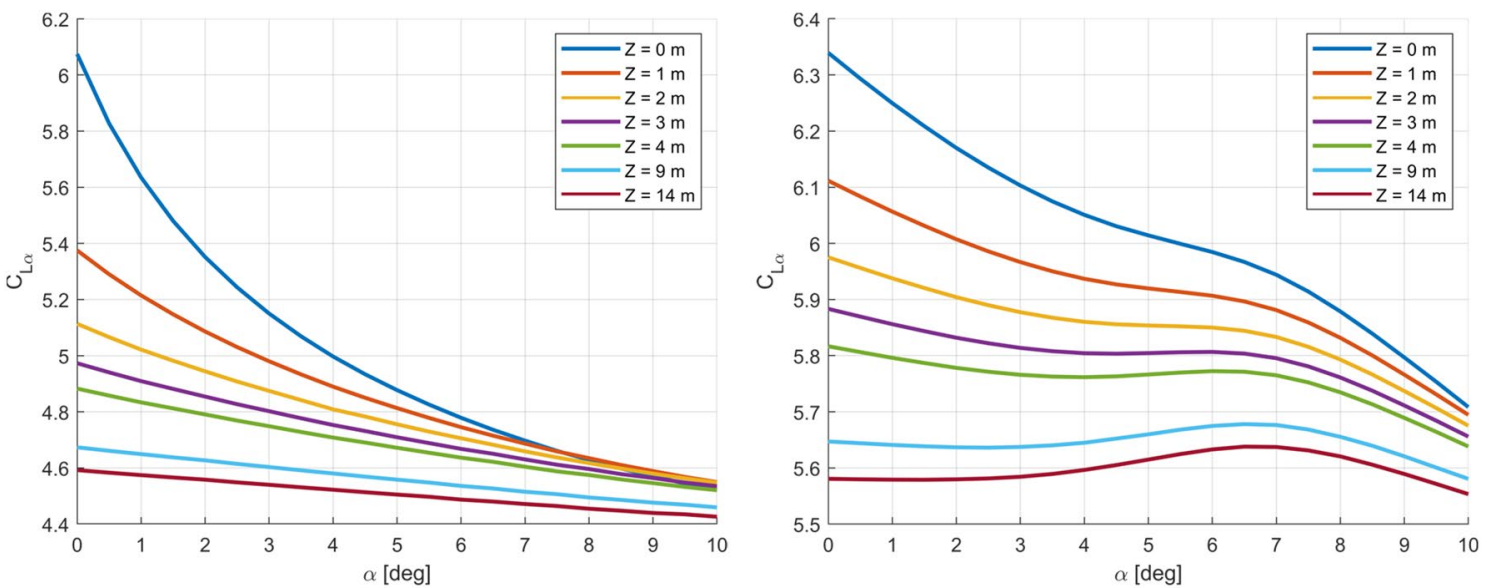

Fig. $10 C_{\mathrm{L} \alpha}$ curves for PrP (left) and CeRAS CSR-01 (right) at clean configuration $\left(\delta_{\mathrm{e}}=\delta_{\mathrm{f}}=0\right)$ 

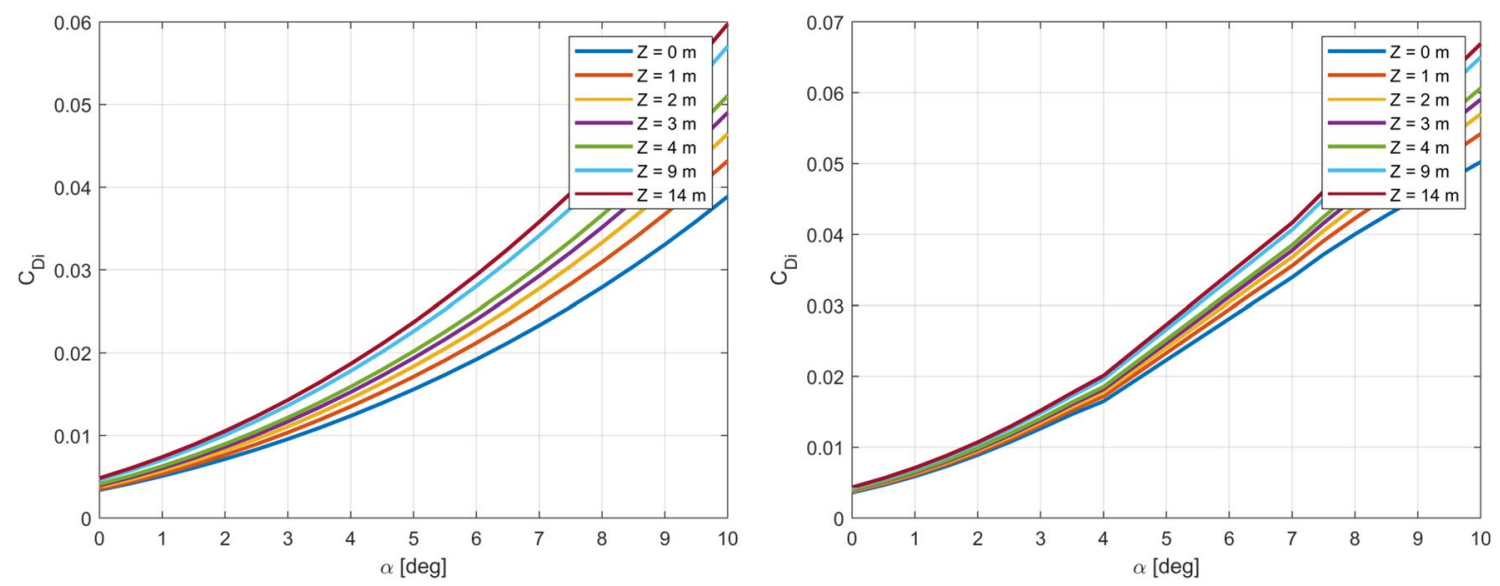

Fig. $11 C_{\mathrm{Di}^{-}}-\alpha$ curves for PrP (left) and CeRAS CSR-01 (right) at clean configuration $\left(\delta_{\mathrm{e}}=\delta_{\mathrm{f}}=0\right)$
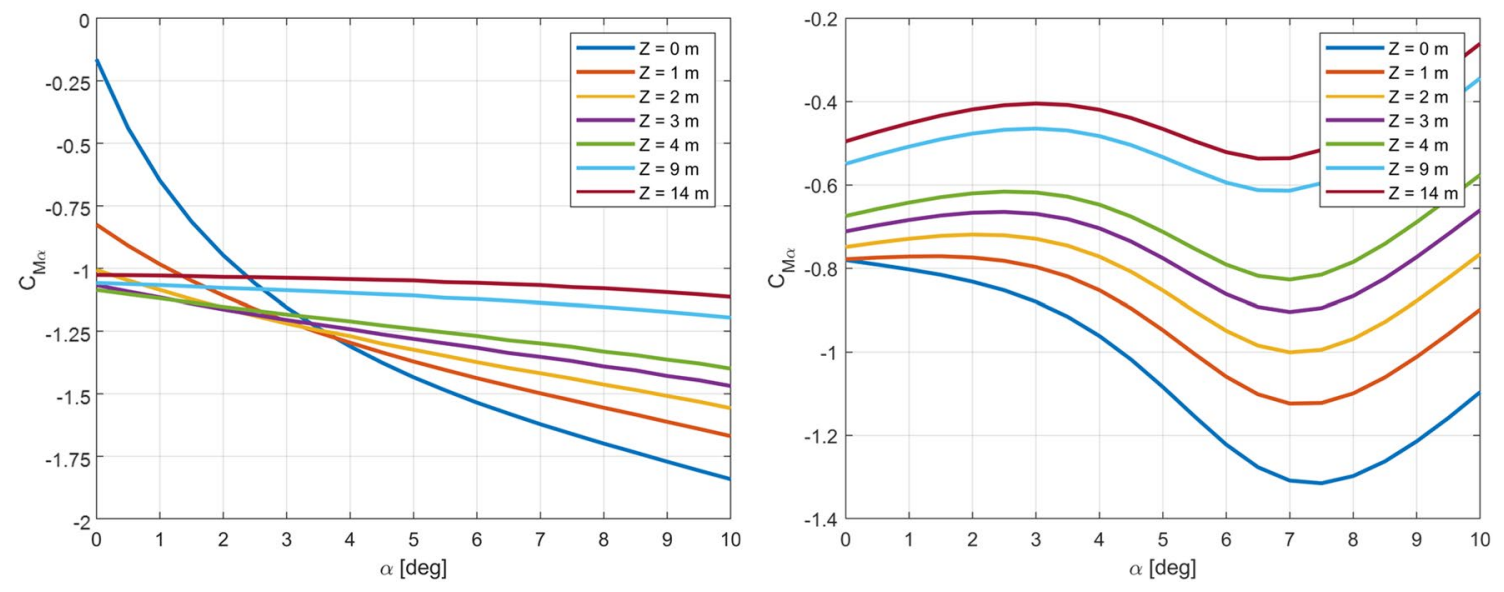

Fig. $12 C_{\mathrm{m} \alpha}-\alpha$ curves for PrP (left) and CeRAS CSR-01 (right) at clean configuration $\left(\delta_{\mathrm{e}}=\delta_{\mathrm{f}}=0\right)$

11,12 , the main aerodynamic coefficients, evaluated with AVL considering the ground effect, are reported for the PrP and CeRAS CSR-01 configurations with respect to $\alpha$ and $z$ variations. The graphs in Fig. 9 show the increase in the lift coefficient as the aircraft approaches the ground.

This is mainly due to the presence of ventral overpressures on the lifting surfaces that causes an increase in the lift generated (effect known as 'air cushion'). This effect becomes more relevant if the distance between the lifting surface and the ground is reduced. For this reason, the PrP configuration has a better advantage from the point of view of lift in ground effect, due to the fact that the front wing, which usually undergoes a higher wing loading than rear wing [32], is much closer to the ground than the main wing of the monoplane competitor. Moving from an altitude of $20 \mathrm{~m}$ to the ground roll condition, and with a zero attitude angle, there is a gain in lift coefficient of $28 \%$ for the PrandtlPlane, while for the conventional monoplane, under the same conditions, there is an increase of $13 \%$. The better performance in ground effect for the Box-Wing is also evident in terms of $C_{\mathrm{L} \alpha}$, as shown in Fig. 10. Compared to the free air flight condition, the PrP configuration has a gain in $C_{\mathrm{L} \alpha}$ equal to $33 \%$ during the ground roll, while for the CERAS CSR-01 it is $14 \%$.

The value of $C_{\mathrm{L} \alpha}$ is not constant with $\alpha$ for configurations that are affected by the ground effect; the graph in Fig. 10 shows that the lifting performance decrease with the increase of aircraft angle of attack: it is due to the distance increase between the wing, and in particular the front wing for Box-Wing, and the runway.

The second significant effect of the proximity of the aircraft to the runway is the reduction of induced drag, as can be seen from the $C_{\mathrm{Di}}$ graphs, shown in Fig. 11. Also for this aspect, the ground effect performance of the PrP is better than the CeRAS CSR-01; moving from free air to 
ground roll, the $C_{\mathrm{L}} / C_{\mathrm{Di}}$ ratio increases by $87 \%$ for the $\operatorname{PrP}$ and $38 \%$ for the CeRAS CSR- 01 .

For the PrP configuration, as the angle of attack increases, such as during the rotation phase in the case of a take-off manoeuvre, the front wing moves away from the ground while the rear wing approaches the ground, causing a pitch stiffening, as can be read from the $C_{\mathrm{m} \alpha}-\alpha$ graph in Fig. 12; with the increase of the distance of the aircraft from the runway this effect tends to disappear. For the monoplane, the greater proximity to the ground causes an increase in pitch stiffness due to the increase in the lift capacity of the main wing.

\section{Simulation Results}

\subsection{Definition of Balanced Field Length}

The evaluation of the Balanced Field Length (BFL) is crucial for the aircraft performance in the take-off phase. According to FAR, two "main distances" are fundamental for the evaluation of the decision speed (balanced $V_{1}$ ) and the BFL: the Take-Off Distance (TOD) and the AccelerateStop Distance (ASD). In this work, the only dry runway is considered.

According to FAR 25.113 the TOD on a dry runway is the greater of:

(a) the horizontal distance $\left(\mathrm{TOD}_{N-1}\right)$ along the take-off path from the start of take-off to the point at which the airplane is 35 feet above the take-off surface. During the take-off path, an engine failure occurs and is recognized at $V_{1}$ (delayed of one-second respect to engine failure speed [36])

b) the $115 \%$ of the horizontal distance $\left(\mathrm{TOD}_{N}\right)$ along the take-off path, with all engine operating, from the start of take-off to the point at which the airplane is 35 feet above the take-off surface.

The first condition is related to a take-off with one engine inoperative (OEI); the second one is related to a take-off with all engine operative (AEO). The above requirement can be expressed as

$\mathrm{TOD}=\max \left\{\mathrm{TOD}_{N-1}, \mathrm{TOD}_{N}\right\}$.

The runway length decreases as the engine failure speed increases; this is due to the fact that an increase of $V_{1}$ allows to reduce the distance covered in the ground-roll phase.

According to FAR 25.109 the ASD on a dry runway is the greatest of:

The sum of the distances $\left(\mathrm{ASD}_{N-1}\right)$ necessary to (a) Accelerate the airplane from a standing start with all engines operating to $V_{\mathrm{EF}}$;

(b) Allow the airplane to accelerate from $V_{\mathrm{EF}}$ until $V_{1}$ with one engine operating;

(c) Come to a full stop plus a distance equivalent to $2 \mathrm{~s}$ at $V_{1}$.

The sum of the distances $\left(\mathrm{ASD}_{N}\right)$ necessary to

(a) Accelerate the airplane from a standing start with all engines operating to $V_{1}$;

(b) Come to a full stop plus a distance equivalent to $2 \mathrm{~s}$ at $V_{1}$.

The above regulation can be expressed as

$\mathrm{ASD}=\max \left\{\mathrm{ASD}_{N-1}, \mathrm{ASD}_{N}\right\}$.

The TOD and the ASD depend on the engine failure speed; in particular, if the engine failure speed increases, the TOD decreases and the ASD increases. At the decision speed $V_{1}$, the TOD is equal to the ASD, and the corresponding runway length is defined Balance Field Length (BFL).

\subsection{Design Parameters: Flaps Deflection, Flap Gain and Take-Off Weight}

The main parameters for the take-off analysis of the PrandtlPlane are flap deflection, take-off weight, and the Flap Gain $\Psi_{\mathrm{f}}$, which is defined as the ratio between the rear wing flap deflection $\left(\delta_{\text {flap-post }}\right)$ and front wing flap deflection $\left(\delta_{\text {flap-ant }}\right)$. Therefore, chosen the front flap deflection among the values $\left[10^{\circ} 20^{\circ} 30^{\circ}\right]$, the rear flap deflection is given by

$\delta_{\text {flap - post }}=\Psi_{\mathrm{f}} \times \delta_{\text {flap - ant }}$.

The rotation speed $V_{\mathrm{R}}$ follows FAR 25.107, according to which $V_{\mathrm{R}}$ has to be higher than $V_{1}$ and has to guarantee the reaching of $V_{2}$. For the CeRAS CSR-01 take-off analysis, all the above parameters are set equal, except for the flap gain, which cannot be defined for a conventional monoplane configuration.

\subsection{Balanced Field Length: Main Results}

In this section, the results of the take-off simulations, in terms of BFL and $V_{1}$, are presented. First of all, the performance of the PrP configuration is discussed; the first parameter analysed is the Flap Gain. In Fig. 13 the curves of the completed take-off manoeuvre (solid lines) and of the aborted take-off manoeuvre (dashed lines) in case of an engine failure at the corresponding speed $V_{\text {failure }}$ are reported. From these results, also reported in the following Table 1 for front wing $\delta_{\text {flap_ant }}=20^{\circ}$ and $\delta_{\text {flap_ant }}=30^{\circ}$, it 


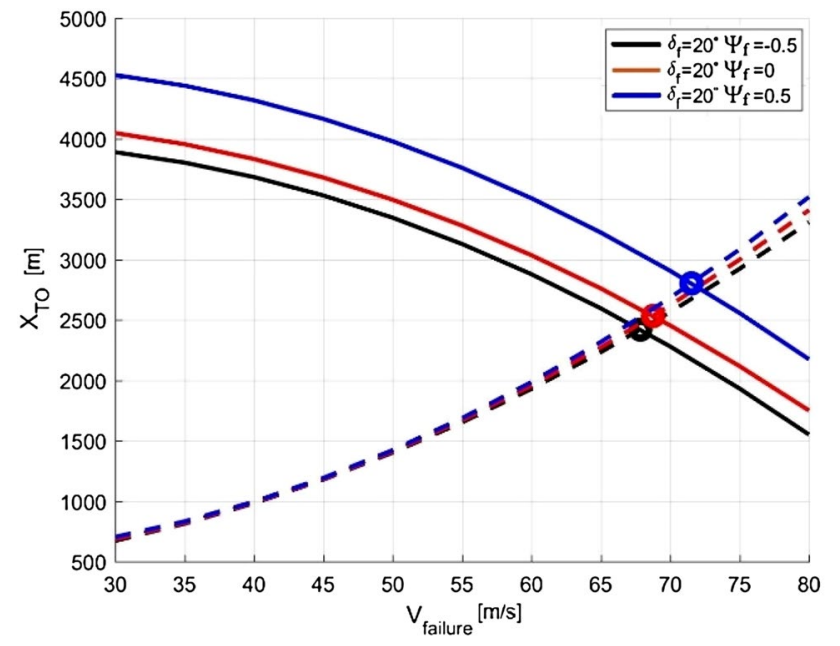

Fig. $13 \mathrm{BFL}$ and $V_{1}$ for the $\operatorname{PrP}$ varying $\Psi_{\mathrm{f}}$ at $\delta_{\text {flap }}=20^{\circ}$

Table 1 BFL and balanced $V_{1}$ for the PrP varying $\Psi_{\mathrm{f}}$

\begin{tabular}{llcll}
\hline$\% W_{\text {TO }}$ & $\delta_{\text {flap_ant }}\left({ }^{\circ}\right)$ & $\Psi_{\mathrm{f}}$ & $V_{1}(\mathrm{~m} / \mathrm{s})$ & BFL $(\mathrm{m})$ \\
\hline 100 & 20 & -0.5 & 67 & 2420 \\
100 & 20 & 0 & 68 & 2530 \\
100 & 20 & 0.5 & 71 & 2810 \\
100 & 30 & -0.5 & 64 & 2180 \\
100 & 30 & 0 & 65 & 2290 \\
100 & 30 & 0.5 & 68 & 2640 \\
\hline
\end{tabular}

clearly follows that increasing the rotation of the rear flap is penalizing in terms of balanced take-off distance while using the rear plain flap in counter-rotation gives a small gain with respect to the case of the clean rear wing.

The reason of these trends relies on the specific architecture and balance of the PrP configuration: giving a positive flap rotation to the rear plain flap increases the pitch down moment that is in contrast to the elevator action necessary for the aircraft rotation (Fig. 14, top-left). The graphs depicted in Fig. 14 show some relevant take-off parameters of a generic PrP take-off manoeuvre without engine failures. Moreover, a positive rear flap rotation increases the total drag force during the ground roll acceleration (Fig. 14, top-right). However, in the following discussion of the results, the reference value of $\Psi_{\mathrm{f}}$ is set equal to zero; negative $\Psi_{\mathrm{f}}$, in fact, may lead to high values of attitude angles $\theta$ (Fig. 14, bottom) that may be incompatible with the tail-clearance requirements for the aircraft during the rotation phase.

The second main parameter that influences the take-off performance is the $\delta_{\text {flap }}$; this parameter, consequently to the definition of the $\Psi_{\mathrm{f}}$ parameter, has to be intended referred to the front wing flap. In the graphs of Fig. 15 are represented the balanced take-off lengths for the PrP configuration, varying the $\delta_{\text {flap }}$ parameter; the same results are reported in Table 2 for two different values of $\Psi_{\mathrm{f}}$. It can be noted that, for both the $\Psi_{\mathrm{f}}$ considered, the runway length requested for the take-off decreases when the $\delta_{\text {flap }}$ increases.

An evaluation of the sensibility on the requested take-off length with respect to the take-off weight has been done; the results are reported in Fig. 16 and in Table 3. The takeoff weights considered are a fraction of the MTOW of the PrP configuration; as expected, the requested balanced field length is shorter for a lighter aircraft.

Following these considerations, the reference setting for the PrP configuration in take-off condition is: $\delta_{\text {flap }}=30^{\circ}$, $\Psi_{\mathrm{f}}=0, W_{\mathrm{TO}}=\mathrm{MTOW}$; this reference configuration is selected to perform a comparison with the take-off performance of the reference monoplane.

For the CeRAS CSR-01 configuration, the evaluation of the performance in take-off condition has been done considering variations of $\delta_{\text {flap }}$ and $W_{\text {TO }}$. As reported in Fig. 17 and Table 4 , increasing the $\delta_{\text {flap }}$ value from $20^{\circ}$ to $30^{\circ}$ does not produce any gain in performance. This may be related to the higher increase in drag coefficient with respect to the increase in lift coefficient for this specific configuration with $\delta_{\text {flap }}=30^{\circ}$.

As described for the PrP configuration, also for the CeRAS CSR-01 reducing the take-off weight implies a reduction in balanced field length. These results are reported in Fig. 18 and Table 5.

The reference setting for the CeRAS CSR-01 configuration in take-off condition is: $\delta_{\text {flap }}=20^{\circ}, W_{\mathrm{TO}}=$ MTOW. Considering the reference PrP setting for the take-off, it is possible to notice that, for the PrP, the BFL is $28.4 \%$ shorter than the BFL requested by the CeRAS CSR-01. This is mainly related to the higher aerodynamic efficiency of the Best Wing System and to the higher aerodynamic gain in ground effect of the PrP configuration.

\subsection{Thrust Reduction}

The above results on the BFL show a shorter runway for the PrP configuration respect to the CeRAS CSR-01; to obtain a comparable runaway length, an engine with a lower thrust-to-weight ratio than the reference value of 0.3 can be installed on the PrP configuration. The thrust model adopted for the take-off simulation and assumed for the previous analyses follows the Eq. 10, as proposed in [37]:

$T=0.75 \frac{5+\mathrm{BPR}}{4+\mathrm{BPR}}\left(T_{\mathrm{MAX}} \cdot N_{\text {engines }}\right)$.

According to this formula, the take-off thrust can be reasonably assumed constant, and its value is about $80 \%$ of the total installed thrust for the considered values of turbofan Bypass Ratio (BPR). In the following analysis, a take-off 

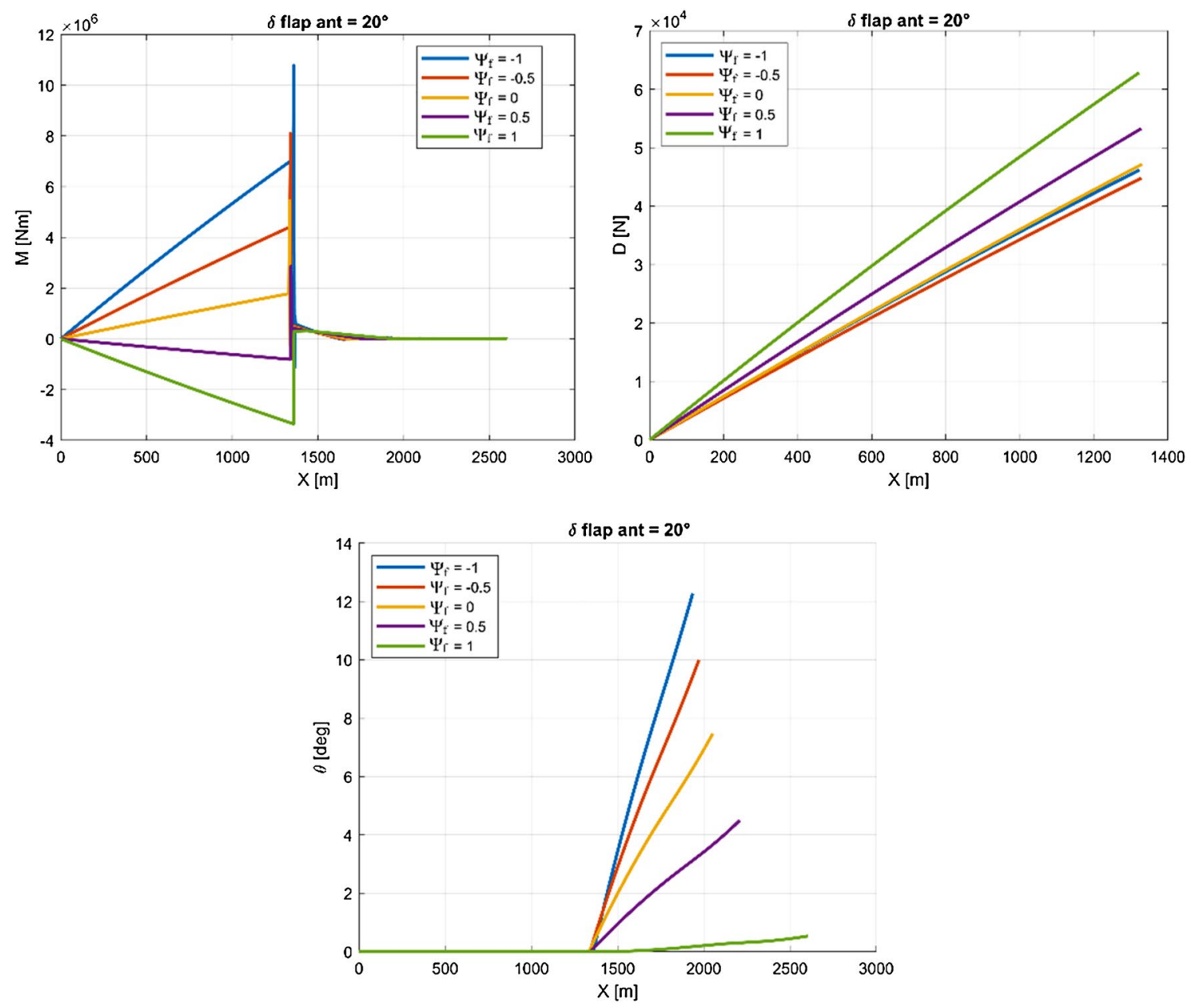

Fig. 14 Pitching moment (top-left), ground roll drag (top-right), attitude (bottom) for a generic take-off manoeuvre for the PrP varying $\Psi_{\mathrm{f}}$

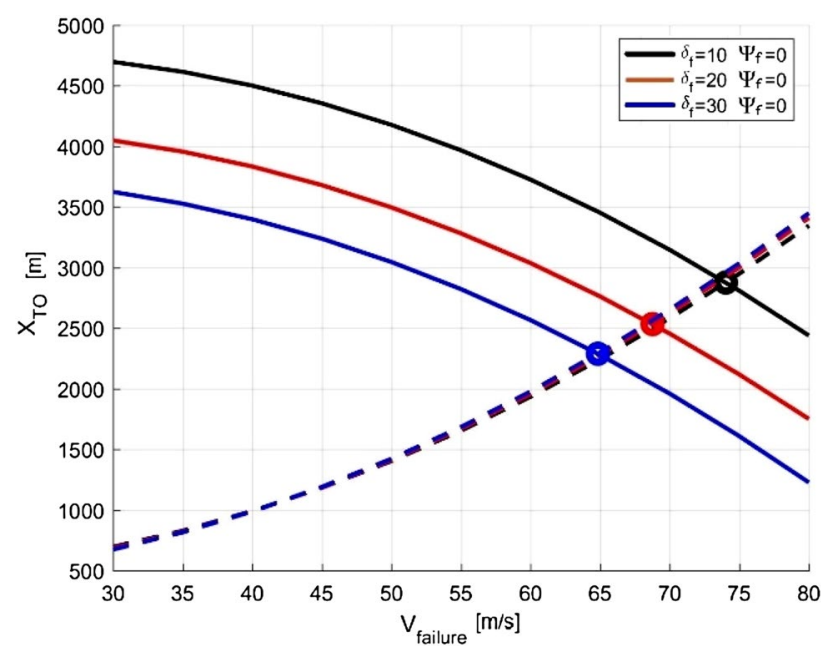

Table 2 BFL and balanced $V_{1}$ for the PrP varying $\delta_{\text {flap }}$

\begin{tabular}{lllll}
\hline$\% W_{\text {TO }}$ & $\delta_{\text {flap_ant }}\left({ }^{\circ}\right)$ & $\Psi_{\mathrm{f}}$ & $V_{1}(\mathrm{~m} / \mathrm{s})$ & BFL $(\mathrm{m})$ \\
\hline 100 & 10 & 0 & 74 & 2870 \\
100 & 20 & 0 & 68 & 2530 \\
100 & 30 & 0 & 64 & 2290 \\
100 & 10 & 0.5 & 75 & 3050 \\
100 & 20 & 0.5 & 71 & 2810 \\
100 & 30 & 0.5 & 68 & 2640 \\
\hline
\end{tabular}

thrust equal to the maximum installed thrust has been considered for the CeRAS CSR-01. The results plotted in Fig. 19 and detailed in Table 6 show a similar performance between the two aircraft; moreover, at maximum flap deflection the BFL, for the PrP, is slightly shorter. Thanks to this preliminary analysis it is possible to consider, for the PrP, an engine thrust reduction of about $20 \%$, ensuring the same performance of the CeRAS CSR-01, in terms of BFL.

Fig. $15 \mathrm{BFL}$ and $V_{1}$ for the $\operatorname{PrP}$ varying $\delta_{\text {flap }}$ for $\Psi_{\mathrm{f}}=0$ 


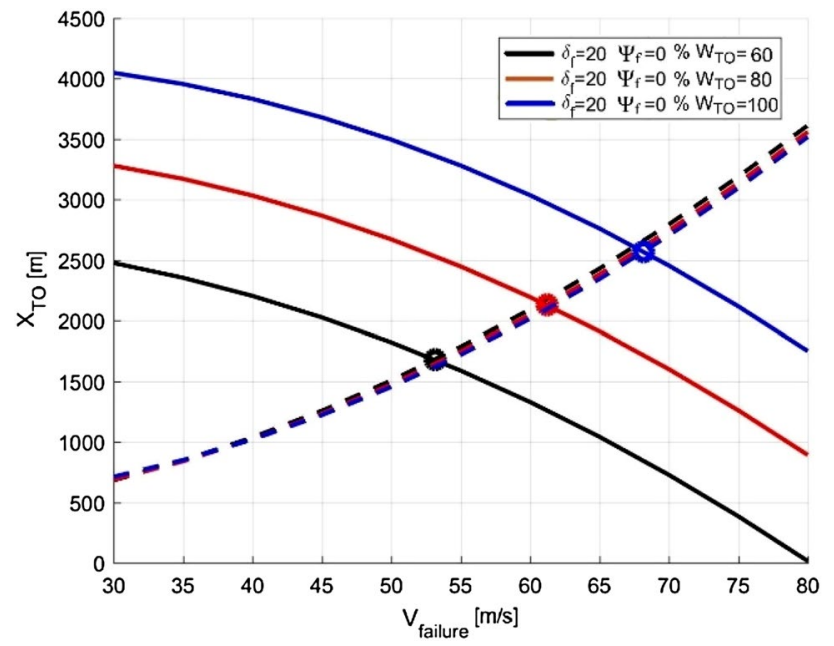

Fig. $16 \mathrm{BFL}$ and $V_{1}$ for the $\operatorname{PrP}$ varying $W_{\mathrm{TO}}$ for $\delta_{\text {flap }}=20^{\circ}$

Table 3 BFL and balanced $V_{1}$ for the PrP varying $W_{\text {TO }}$

\begin{tabular}{lllll}
\hline$\% W_{\text {TO }}$ & $\delta_{\text {flap_ant }}\left({ }^{\circ}\right)$ & $\Psi_{\mathrm{f}}$ & $V_{1}(\mathrm{~m} / \mathrm{s})$ & BFL $(\mathrm{m})$ \\
\hline 60 & 20 & 0 & 53 & 1680 \\
80 & 20 & 0 & 61 & 2130 \\
100 & 20 & 0 & 68 & 2530 \\
60 & 30 & 0 & 51 & 1520 \\
80 & 30 & 0 & 58 & 1920 \\
100 & 30 & 0 & 64 & 2290 \\
\hline
\end{tabular}

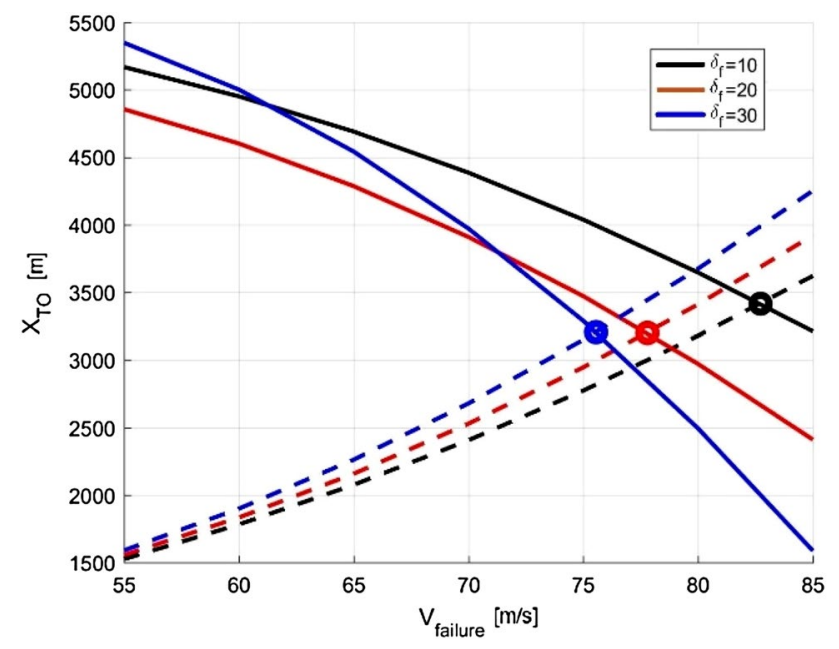

Fig. $17 \mathrm{BFL}$ and $V_{1}$ for the CeRAS CSR-01 varying $\delta_{\text {flap }}$

\subsection{Standard Take-Off: Numerical Results}

In this section, the results of take-off simulations in standard conditions, without engine failures, are presented. The conditions set for PrP and CeRAS CSR-01 simulations are:
Table 4 BFL and balanced $V_{1}$ for the CeRAS CSR-01 varying $\delta_{\text {flap }}$

\begin{tabular}{llll}
\hline$\% W_{\text {TO }}$ & $\delta_{\text {flap_ant }}\left({ }^{\circ}\right)$ & $V_{1}(\mathrm{~m} / \mathrm{s})$ & BFL $(\mathrm{m})$ \\
\hline 100 & 10 & 82 & 3410 \\
100 & 20 & 77 & 3200 \\
100 & 30 & 75 & 3210 \\
\hline
\end{tabular}

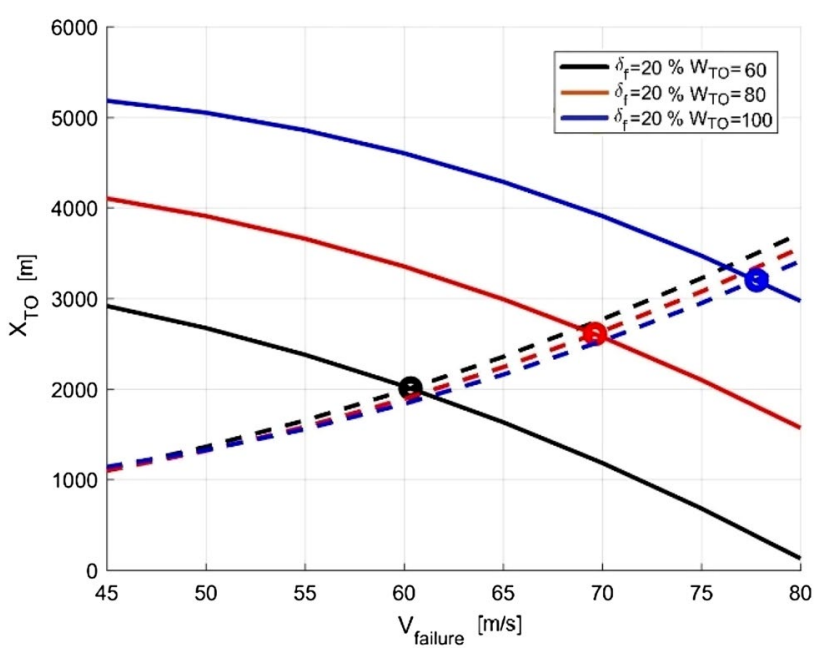

Fig. $18 \mathrm{BFL}$ and $V_{1}$ for the CeRAS CSR-01 varying $W_{\mathrm{TO}}$

Table 5 BFL and balanced $V_{1}$ for the CeRAS CSR-01 varying $W_{\text {TO }}$

\begin{tabular}{llll}
\hline$\% W_{\text {TO }}$ & $\delta_{\text {flap_ant }}\left({ }^{\circ}\right)$ & $V_{1}(\mathrm{~m} / \mathrm{s})$ & BFL $(\mathrm{m})$ \\
\hline 60 & 20 & 60 & 2005 \\
80 & 20 & 69 & 2610 \\
100 & 20 & 77 & 3200 \\
60 & 30 & 58 & 2010 \\
80 & 30 & 67 & 2610 \\
100 & 30 & 75 & 3210 \\
\hline
\end{tabular}

1. Full thrust for the two engines for the entire manoeuvre;

2. Dry runway;

3. No wind during the take-off;

4. Airport at sea level;

5. Standard air condition.

In this section, the influence of the main parameters $\left(\delta_{\text {flap }}\right.$ and $\Psi_{\mathrm{f}}$ ) is presented for the PrP configuration; no flap gain was considered for the CeRAS CSR-01. To evaluate the aircraft performance, three main quantities are considered: pitch angle at the end of the take-off, average vertical load factor $\left(n_{\mathrm{z}}\right)$ during the lift-off phase, for the passenger comfort, and the runway length; all the data are evaluated at MTOW. 


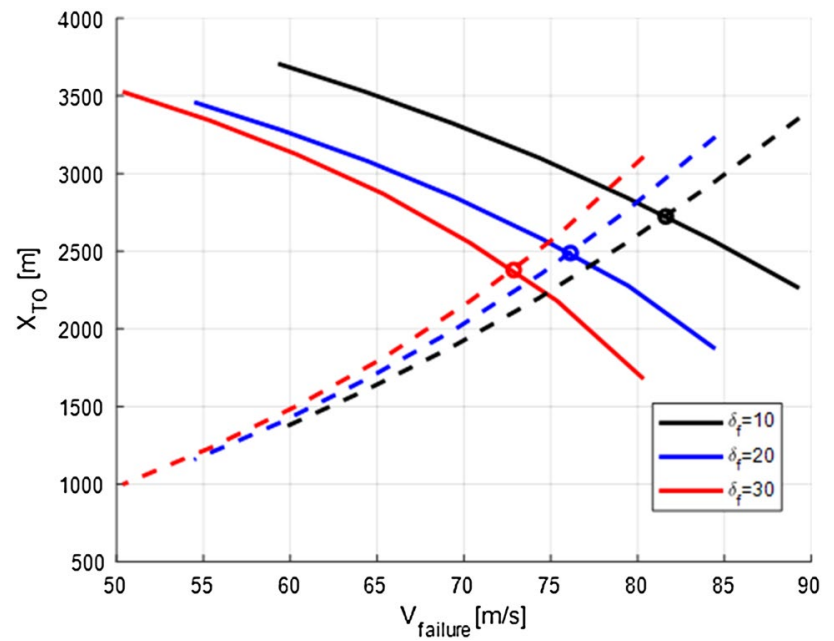

Fig. $19 \mathrm{BFL}$ and $V_{1}$ for the CeRAS CSR-01 varying flap deflection at $T / W=0.3$

Table 6 BFL and $V_{1}$ for the CeRAS CSR-01 varying flap deflection at $\mathrm{T} / \mathrm{W}=0.3$

\begin{tabular}{llll}
\hline$\% W_{\text {TO }}$ & $\delta_{\text {flap_ant }}\left({ }^{\circ}\right)$ & $V_{1}(\mathrm{~m} / \mathrm{s})$ & BFL $(\mathrm{m})$ \\
\hline 100 & 10 & 82 & 2724 \\
100 & 20 & 76 & 2489 \\
100 & 30 & 72 & 2379 \\
\hline
\end{tabular}

Table 7 Vertical load factor and runway length comparison at $\Psi_{\mathrm{f}}=0$

\begin{tabular}{lll}
\hline$\delta_{\text {flap }}\left(^{\circ}\right)$ & $\Delta n_{\mathrm{z}}(\%)$ & $\begin{array}{l}\Delta \text { Runway } \\
\text { length }(\%)\end{array}$ \\
\hline 10 & -5.2 & -11.6 \\
20 & -4.3 & -12.7 \\
30 & -2.6 & -15.1 \\
\hline
\end{tabular}

Fig. 20 BFL and TOD of PrP and CeRAS CSR-01 varying $\delta_{\text {flap }}$
For the PrP, reducing the flap gain causes an increase in the vertical load factor. It is reasonable to assume that this trend is due to the PrP architecture; a flap deflection of the rear wing increases the pitching moment, and a lower angle of attack is developed, so lower lifting force and, consequently, lower $n_{\mathrm{z}}$. The results for the CeRAS CSR01 take-off show no influence of flap deflection on $n_{\mathrm{z}}$ and a slight influence on final pitch angle. The comparison, in percentual terms, is detailed in Table 7; in this case, the PrP flap gain is set equal to zero.

The results comparison, considering the reference flaps settings for the two configurations, shows a shorter runway length, a lower vertical load factor (resulting in an increase of the passenger comfort), and higher pitch angle for the PrP; the results in terms of BFL, TOD, $n_{\mathrm{z}}$ and $\theta$, varying $\delta_{\mathrm{f}}$ and $\Psi_{\mathrm{f}}$, are reported in Figs. 20, 21, 22.

Considering a flap deflection of $20^{\circ}$ (and a zero $\Psi_{\mathrm{f}}$ for the PrP), a trajectory comparison is depicted in Fig. 23.

\section{Conclusions}

In this paper, the preliminary take-off performance analysis of a PrandtlPlane aircraft have been presented, together with a comparison between the take-off characteristics of a PrandtlPlane and a reference monoplane. The configurations selected for the comparison are the baseline PrandtlPlane aircraft developed in the framework of the PARSIFAL Project, and the reference monoplane CeRAS CSR-01. The simulation of take-off dynamics has been carried out by means of an in-house tool, which numerically integrates the equation of motion, taking the ground effect into account. From the preliminary analysis of the aerodynamic results considering the ground effect, it emerges that the PrandtlPlane has a higher gain on $C_{\mathrm{L}}$,
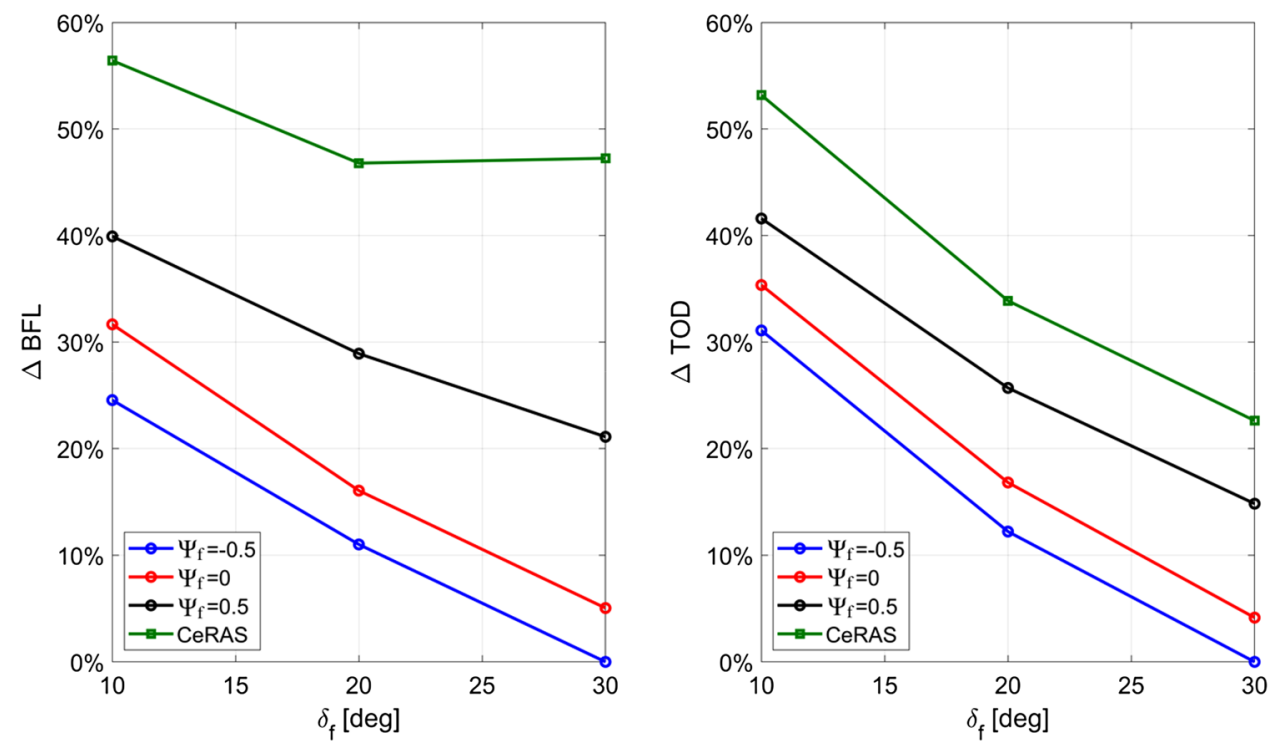


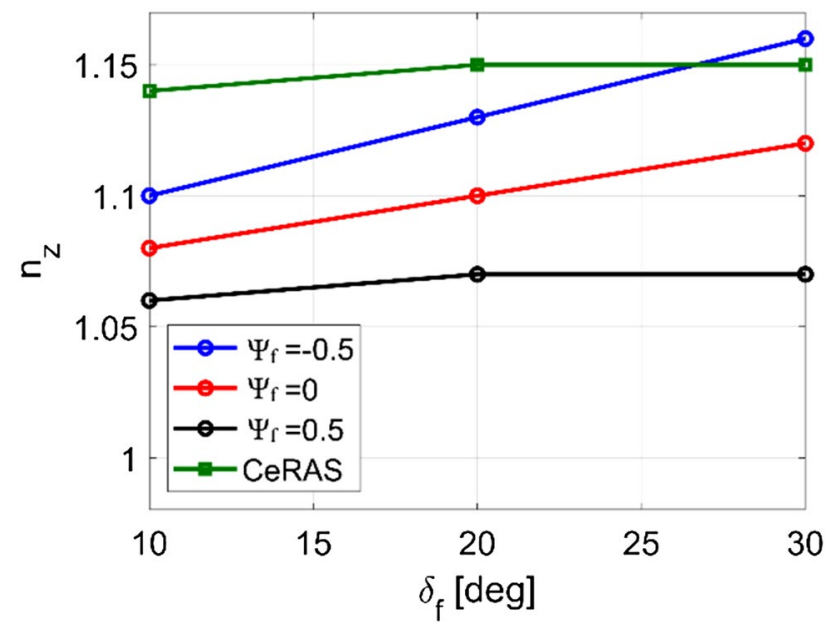

Fig. 21 Vertical load factor of PrP and CeRAS CSR-01 varying $\delta_{\text {flap }}$

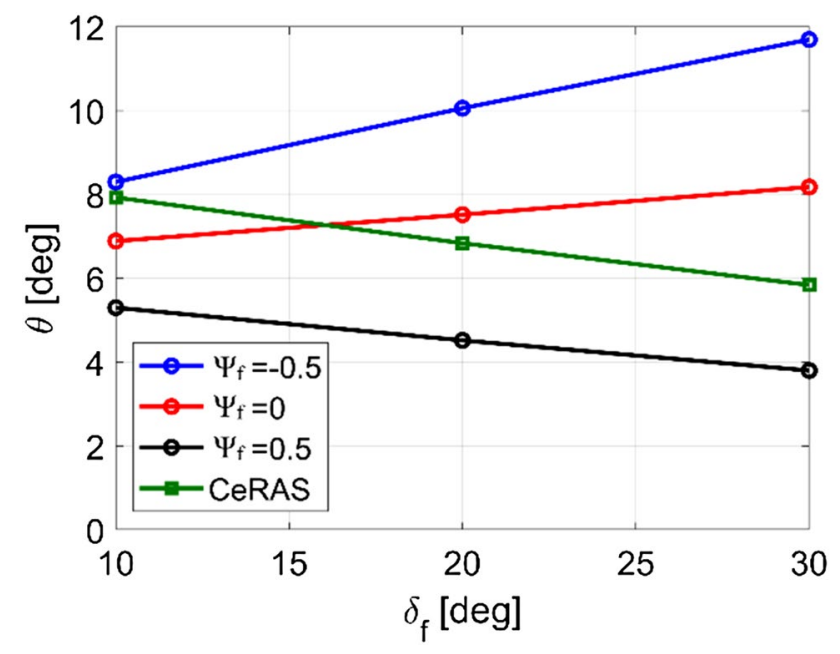

Fig. 22 Attitude of PrP and CeRAS CSR-01 at the end of the take-off varying $\delta_{\text {flap }}$

$C_{\mathrm{L} \alpha}$, and aerodynamic efficiency, with respect to the competitor monoplane. The reason behind this behaviour is strictly related to its architecture: the front wing is very close to the ground and is, therefore, more affected by the ground effect. The analysis of the simulations of the takeoff shows also that the reference PrandtlPlane aircraft has better take-off performance with respect to the conventional competitor, in terms of take-off runway length and passengers comfort. However, the results here presented are preliminary and can be improved; in the future activities, a calibration of the low-fidelity ground effect aerodynamic evaluation will be carried out, using RANS models.

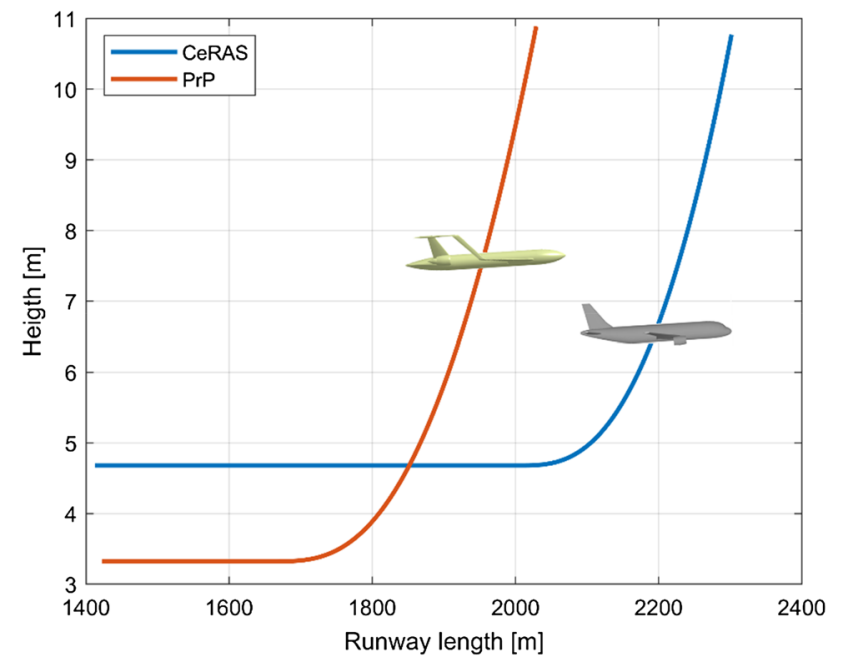

Fig. 23 Centre of gravity trajectory comparison between $\operatorname{PrP}$ and CeRAS CSR-01

Then, a surrogate model will be built, to strongly reduce the computational time for each take-off simulation; in this way, a wider set of design parameters can be analysed, and optimization procedures for take-off manoeuvre can be set up.

Acknowledgements Open access funding provided by University of Pisa within the CRUI-CARE Agreement. The present paper concerns part of the activities carried out within the research project PARSIFAL ("Prandtlplane ARchitecture for the Sustainable Improvement of Future AirpLanes"), which has been funded by the European Union under the Horizon 2020 Research and Innovation Program (Grant agreement n.723149).

\section{Compliance with Ethical Standards}

Conflict of Interest On behalf of all authors, the corresponding author states that there is no conflict of interest.

Open Access This article is licensed under a Creative Commons Attribution 4.0 International License, which permits use, sharing, adaptation, distribution and reproduction in any medium or format, as long as you give appropriate credit to the original author(s) and the source, provide a link to the Creative Commons licence, and indicate if changes were made. The images or other third party material in this article are included in the article's Creative Commons licence, unless indicated otherwise in a credit line to the material. If material is not included in the article's Creative Commons licence and your intended use is not permitted by statutory regulation or exceeds the permitted use, you will need to obtain permission directly from the copyright holder. To view a copy of this licence, visit http://creativecommons.org/licenses/by/4.0/.

\section{References}

1. Airbus, Global Market Forecast 2017-2037, Airbus Commercial Aircraft, Toulouse (2017) 
2. Boeing, Global Market Outlook 2017-2036, Boeing Commercial Airplanes, Seattle (2017)

3. PARSIFAL Consortium: Report on socio-economic scenarios and expectations. Deliverable 1.1 (2018). http://www.parsifalproject .eu

4. Lee, D.S., et al.: Aviation and global climate change in the $21 \mathrm{st}$ century. Atmos. Environ. 43, 3520-3537 (2009). https://doi. org/10.1016/j.atmosenv.2009.04.024

5. Schäfer, W.A., et al.: Technological, economic, and environmental prospect of all-electric aircraft. Nature Energy 4, 160-166 (2019). https://doi.org/10.1038/s41560-018-0294-x

6. ACARE: Strategic Research and Innovation Agenda (SRIA), Advisory Council for Aviation Research and Innovation in Europe (2017). http://www.acare4europe.org

7. Cavallaro, R., Demasi, L.: Challenges, ideas, and innovations of joined-wing configurations: a concept from the past, an opportunity for the future. Prog. Aerosp. Sci. (2016). https://doi. org/10.1016/j.paerosci.2016.07.002

8. Liebeck, R.H.: Design of the blended wing body subsonic transport. J. Aircr. (2004). https://doi.org/10.2514/1.9084

9. Gern, F.H., et al.: Multidisciplinary design optimization of a transonic commercial transport with a strut-braced wing. In: AIAA, World Aviation Conference, San Francisco (1999). https://doi. org/10.4271/1999-01-5621

10. Frediani, A.: Swept-wing box-type aircraft with high flight static stability. European patent N. EP1597145 (2005)

11. Prandtl, L.: Induced drag of multiplanes. Technical Report TN 182, NACA (1924)

12. Frediani, A., Montanari, G.: Best Wing System: An Exact Solution of the Prandtl's Problem, Variational Analysis and Aerospace Engineering. Springer, Berlin (2009)

13. Gall, P.D., Smith, H.C.: Aerodynamic characteristics of biplanes with winglets. J. Aircr. (1987). https://doi.org/10.2514/3.45470

14. Frediani, A.: The Prandtl Wing. VKI Lecture Series: Innovative Configurations and Advanced Concepts for Future Civil transport Aircraft (2005)

15. Gagnon, H., Zingg, D.W.: Aerodynamic optimization trade study of a Box-Wing aircraft configuration. J. Aircr. (2016). https://doi. org/10.2514/1.C033592

16. Demasi, L., Dipace, A., Monegato, G., Cavallaro, R.: Invariant formulation for the minimum induced drag conditions of nonplanar wing systems. AIAA J. (2014). https://doi.org/10.2514/1. J052837

17. Frediani, A., Cipolla, V., Rizzo, E.: The PrandtlPlane configuration: overview on possible applications to civil aviation. In: Buttazzo, G., Frediani, A. (eds.) Variational Analysis and Aerospace Engineering: Mathematical Challenges for Aerospace Design. Springer Optimization and Its Applications, vol. 66. Springer, Boston (2012). https://doi.org/10.1007/978-1-4614-2435-2_8

18. Frediani, A., Cipolla, V., Oliviero, F.: Design of a prototype of light amphibious PrandtlPlane. In: 56th AIAA/ASCE/AHS/ASC Structures, Structural Dynamics, and Materials Conference, AIAA SciTech Forum (2015). https://doi.org/10.2514/6.2015-0700

19. Andrews, A.S., Perez, R.E.: Multidisciplinary analysis of a BoxWing aircraft designed for a regional-jet mission. In: 16th AIAA/ ISSMO Multidisciplinary Analysis and Optimization Conference, Dallas, 2015. https://doi.org/10.2514/6.2015-2793https:// doi.org/10.2514/6.2015-2793
20. PARSIFAL Consortium: Requirements for the adoption of the PrandtlPlane as a mean of transport. Deliverable 2.1, PARSIFAL Project (2018). http://www.parsifalproject.eu

21. Pistolesi, E.: Il Problema dell'ala in Vicinanza del Suolo, L'Aerotecnica, vol. 13 (1933)

22. Pistolesi, E.: Ground effect-theory and practice. NACA TM 828 (1937)

23. CeRAS - Central Reference Aircraft System. http://www.ceras.ilr. rwthaachen.de/trac/wiki/CeRAS/AircraftDesigns/CSR01

24. Risse, K., Schäfer, K., Schültke, F., Stumpf, E.: Central Reference Aircraft data System (CeRAS) for research community. CEAS Aeronaut. J. (2016). https://doi.org/10.1007/s13272-015-0177-9

25. Risse, K., Anton, E., Lammering, T., Franz, K.: An integrated environment for preliminary aircraft design and optimization. In: 8th AIAA Multidisciplinary Design Optimization Specialist Conference, AIAA, Honolulu (2012). https://doi. org/10.2514/6.2012-1675

26. Frediani, A., Cipolla, V., Abu Salem, K., Binante, V., Scardaoni Picchi, M.: Conceptual design of PrandtlPlane civil transport aircraft. Proc. Inst. Mech. Eng. Part G J. Aerosp. Eng. (2019). https ://doi.org/10.1177/0954410019826435

27. Cipolla, V., Frediani, A., Abu Salem, K., Picchi Scardaoni, M., Nuti, A, Binante, V.: Conceptual design of a box wing aircraft for the air transport of the future. In: AIAA Aviation Forum (2018). https://doi.org/10.2514/6.2018-3660

28. Abu Salem, K., Binante, V., Cipolla, V., Maganzi, M.: PARSIFAL project: a breakthrough innovation in air transport. Aerotecnica Missili Spazio (2018). https://doi.org/10.19249/ams.v97i1.337

29. Abu Salem, K., Cipolla, V., Carini, M., Méheut, M., Kanellopoulos, S., Binante, V., Maganzi, M.: Aerodynamic design and preliminary optimization of a commercial PrandtlPlane aircraft. In: 8th EUCASS conference, Madrid (2019)

30. Drela, M.: AVL code. http://web.mit.edu/drela/Public/web/avl/

31. Torenbeek, E.: Synthesis of subsonic airplane design. Springer, Berlin (1982)

32. Abu Salem, K., Palaia, G., Cipolla, V., Binante, V, Zanetti, D.: Aerodynamic design procedures and tools for box-wing transport aircraft. In: Aerospace European Conference, Bordeaux (2020)

33. Bianchi, M.: Analysis of take-off performance of a Prandtlplane 300 seat civil transport aircraft. Master Thesis, University of Pisa (2018)

34. Fink, M.P., Lastinger, J.L.: Aerodynamic characteristics of lowaspect-ratio wings in close proximity to the ground. NASA Technical Note (1961)

35. Mantle, P.J.: Induced drag of wings in ground effect. Aeronaut. J. (2016). https://doi.org/10.1017/aer.2016.106

36. Airbus: Getting to grips with aircraft performance (2002)

37. Casarosa, C.: Meccanica del volo. Plus-Pisa University Press, Pisa (2004)

Publisher's Note Springer Nature remains neutral with regard to jurisdictional claims in published maps and institutional affiliations. 